\title{
Use Electrons Sparingly but Efficiently, the Battle to get All the Required Information Needed While Minimizing Dose and Maximizing Data Collection at the Highest Resolution.
}

\author{
Jan Ringnalda ${ }^{1}$, Arda Genc ${ }^{1}$, Eric Van Capellen ${ }^{1}$ \\ ${ }^{1 .}$ FEI Company, 5350 NE Dawson Creek Dr., Hillsboro, OR 97124
}

Since aberration correction has been applied on modern electron microscope systems, there has been a need to demonstrate the benefits of this capability and in some respect, to justify the cost of these complex systems. Sometimes the justification of such a system is by the presentation of a colorful elemental map which correlates with the atomic periodicity in the sample. This type of visualization, while artistic, may not be sufficient to characterize materials at the levels proclaimed, since there are many events happening which are difficult to place with atomic certainty. There are many benefits of the correctors on the imaging side of the sample by removing delocalization and improving the image interpretability for phase contrast imaging, however there is a strict requirement on the sample both in terms of cleanliness, thickness and damage layers. In the case of correctors on the condenser or probe forming part of the microscope, this requirement is only amplified especially when the imaging techniques are combined with various spectroscopies.

There are many aspects of the experiment to consider when looking at the effects of a probe corrector, for instance: The size of the real volume analyzed and the amount of current that has been put into a very small area. Besides the geometrical aspects of convergence angle increase [1] and the resultant effective increase of the analytical footprint through amorphous or glassy materials, for crystalline materials there is the channeling/de-channeling problem that will convolute any attempt to get real quantified data from samples at the scales required - or promised. The ability to change convergence angle to suit the analytical requirements will enable scientists to optimize the experiment, however it will take some experience to establish what the optimum experimental setup will be for a given experiment. And then there is the sample, will it be stable under the beam, will it remain in a state representative of the bulk it came from during preparation, and will the inspection using a high intensity electron beam not change the material during investigation?

To be clear, any sample put into a modern electron microscope system can be damaged. Our colleagues in the life sciences have long been using dose mitigation techniques and cooling samples to extend the life of the sample under the beam. As life science and materials science get closer together, materials scientists can learn something regarding cryogenic work, image analysis and dose mitigation. Sub nanometer particles tend to move or disappear before they can be analyzed. It was long thought that carbonaceous materials would not damage at $80 \mathrm{kV}$. Once we put a corrected probe on graphene at $80 \mathrm{kV}$ we soon learned otherwise. So the HT was dropped to $60 \mathrm{kV}$. Still samples can be damaged at that accelerating voltage so now we are looking at $40 \mathrm{kV}$, and the quest remains high resolution imaging coupled with analysis.

This paper will show some of the recent high resolution data that has been obtained with a system capable of $300 \mathrm{kV}$ work but has been operated at $40 \mathrm{kV}$. Image resolution in both TEM and STEM mode will be discussed, and limitations will be highlighted, also sample requirements will be shown. This 
type of sample can only really be generated using a specialized dualbeam system capable of effective low $\mathrm{kV}$ cleaning or perhaps a perfect sample as provided by nature in the shape of 2D materials. Especially if the sample in question has to be inspected to reveal detail in three dimensions [2], it will have to be inspected for extended periods of time through many tilt orientations.

The key is being efficient with the electrons, so using a detector that is most efficient and gives the data needed. In terms of imaging, especially STEM imaging, the HAADF technique is not very efficient and has problems when trying to image light elements. ABF is more efficient however there can be focus effects and thickness effects rather like in phase contrast TEM techniques, so care must be taken to interpret the data. The relatively new iDPC imaging technique from FEI is more efficient than ABF and does not suffer from the focus or thickness issues like the ABF technique, and is suitable for imaging light elements in the correct atomic positions. Figure 1 shows data from a Silicon sample shot at $40 \mathrm{keV}$ on a Titan system. Figure 2 shows an image acquired using the iDPC technique, the new, more efficient ABF imaging technique from FEI.

Figure 1. Image of Si taken at $40 \mathrm{keV}$ in STEM.

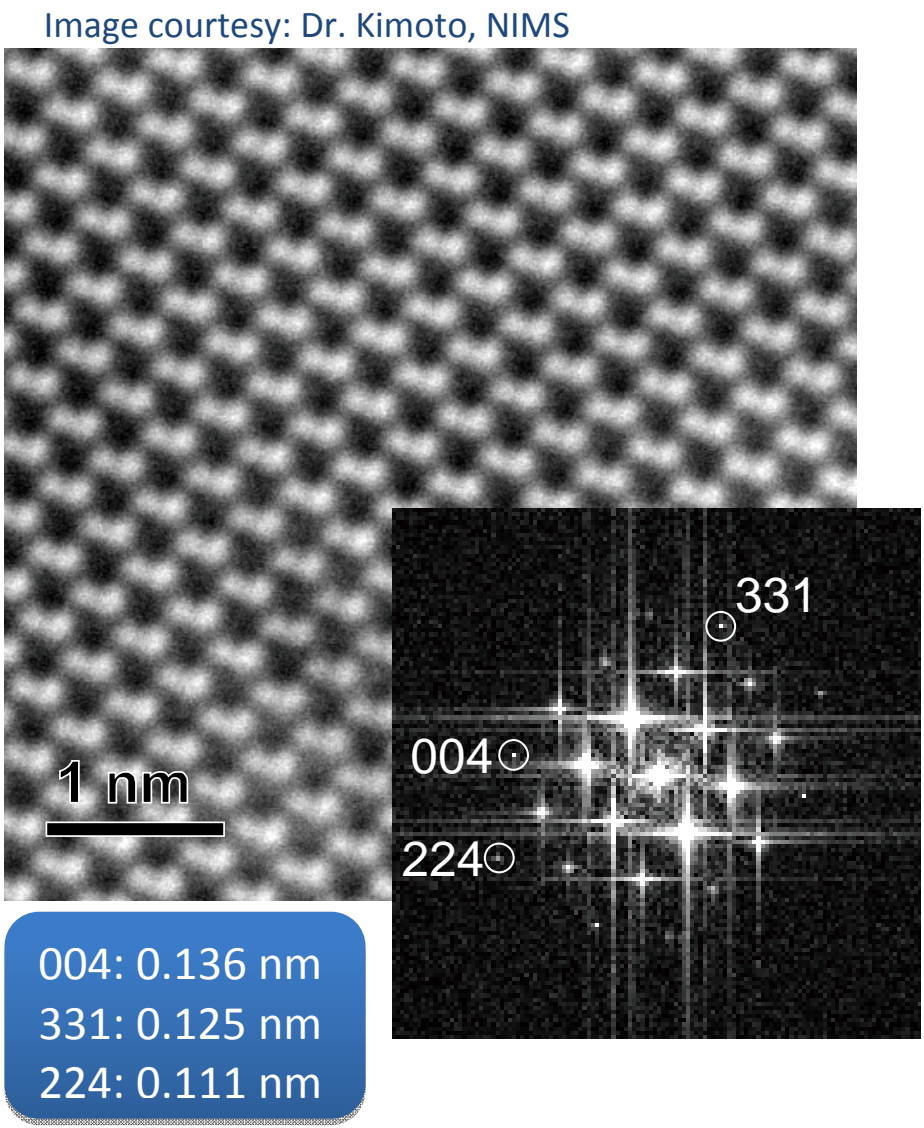

References:

[1] J. Bruley et al, M\&M conference proceedings 2010 [2] A. Genc et al, M\&M Conference Proceedings 2014
Figure 2. Comparison between ADF and iDPC on graphene. Both images were acquired simultaneously with the same dose.

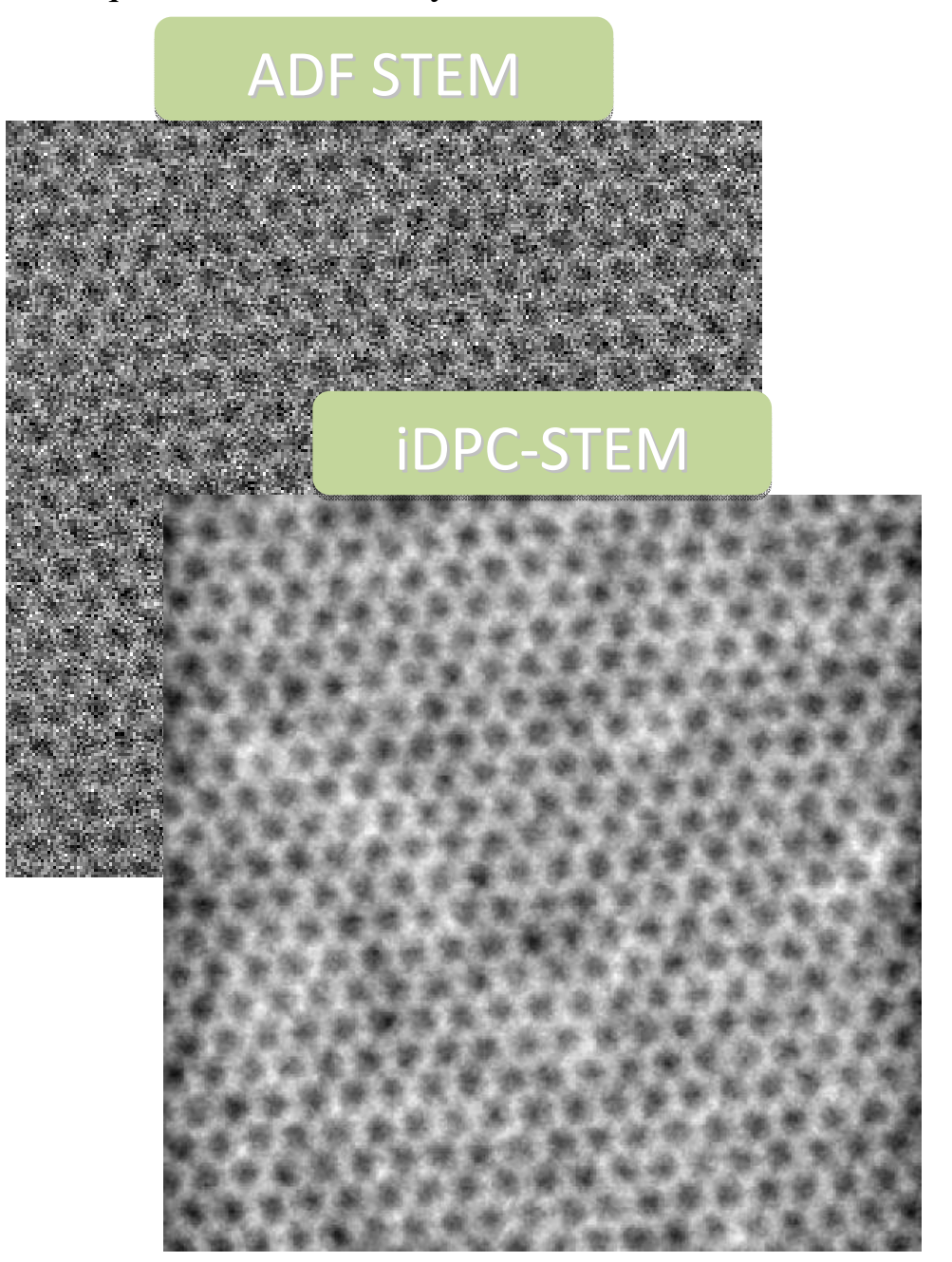

